\title{
Lokalanästhesie am Kopf beim Heimtier
}

Stefan Gabriel

\author{
Während bei Hund und Katze die Leitungsanästhesie weit verbreitet ist, wird sie bei \\ Heimtieren oft vergessen. Dabei ist die Lokalanästhesie im Rahmen einer multi- \\ modalen Schmerztherapie wegen der fehlenden Analgesie von Isofluran und nach \\ Antagonisierung der Triple-Narkose wichtig für die Rekonvaleszenz. Denn insbeson- \\ dere herbivore Zahnpatienten müssen postoperativ schnellstmöglich wieder fressen.
}

\section{Einleitung}

Die Vorgehensweise ist bei Heimtieren grundsätzlich analog zu der bei Hund und Katze. Durch Infiltration oder Leitungsblock wird die Schmerzübermittlung an höhere Zentren unterbrochen, wodurch die zentrale Schmerzrezeption und das Schmerzgedächtnis verhindert werden. Einschränkungen erfährt die Verwendung der Lokalanästhesie bei kleinen Heimtieren durch technische Probleme bei der Applikation und durch die bei geringen Körpergewichten relativ geringe therapeutische Dosis.

\footnotetext{
Merke

Zur Vermeidung von Nebenwirkungen muss die intravasale Injektion sicher ausgeschlossen werden und die toxische Dosis ( $2 \mathrm{mg} / \mathrm{kg} \mathrm{KGW}$ bei Bupivacain und 5 mg/kg KGW bei Lidocain) darf nicht erreicht werden.
}

Fehlende Treffergenauigkeit bei der Applikation kann bei kleinen Heimtieren leider nicht wie beim Großtier durch Verwendung großer Injektionsvolumina ausgeglichen werden. Das Risiko der intravasalen Injektion besteht bei jeder Leitungsanästhesie, weil periphere Nerven, zumal in Knochenkanälen des Schädels, stets von Gefäßen begleitet werden.

Die Anforderung an die Injektionstechnik ist also die Verwendung einer möglichst dünnen (atraumatischen) Nadel, Begrenzung des Injektionsvolumens und möglichst Aspiration nach Platzierung der Nadel.

Arzneimittelrechtlich ist kein Lokalanästhetikum für kleine Heimtiere (minor species) zugelassen. Es muss deshalb stets eine Umwidmung von Veterinärpräparaten (3. Stufe der Kaskade) oder Humanpräparaten (4. Stufe) vorgenommen werden. Von der Verwendung von Präparaten mit Sperrkörper (Adrenalin) wird abgeraten. Diese haben zwar den Vorteil einer verlängerten Wirkdauer und reduzierter Gewebsblutung, bei irrtümlicher intrava- saler Verschleppung sind jedoch kardiale Nebenwirkungen (Arrhythmien) zu befürchten. Das am häufigsten verwendete Lokalanästhetikum ist Lidocain (Wirkungsdauer 60-120 Min.). Es eignet sich zur Infiltration und Nervenblockade. Gleichfalls geeignet ist Bupivacain aus der Humanmedizin (Wirkungsdauer 180-360 Min.).

\section{LIDOCAIN}

Kalkulation der maximal anwendbaren Dosis zur

Vermeidung toxischer Effekte:

- maximal empfohlene Dosis: $5 \mathrm{mg} / \mathrm{kg}$

- bei $2 \%$ iger Lösung sind das: 0,25 ml/kg KGW

Zum Vergleich:

- LD $_{50}$ Kaninchen i.v.: $26-40 \mathrm{mg} / \mathrm{kg}$

- LD 50 Kaninchen s. c.: 200-460 mg/kg

- LD $_{100}$ Kaninchen i. v.: 59,3 mg/kg (aus diverser Literatur)

\section{Injektionstechnik}

Die Verwendung klassischer Injektionskanülen und Einmalspritzen erweist sich wegen des großen Totraums und des unkontrollierbaren Injektionsdrucks bei kleinen Heimtieren als problematisch. Die Applikation erfolgt entweder sehr vorsichtig mit einer Tuberkulinspritze und dünner Kanüle ( $\triangleright$ Abb. 1), z. B. 23 G 0,6 616 mm, in der man nicht mehr als die maximal geplante Injektionsmenge aufzieht. Besonders wichtig sind der feste Sitz der Kanüle auf dem Konus und das vorherige Ausdrücken von Luftbläschen.

\section{Merke}

Vor der mit nur leichtem Druck auszuführenden Injektion prüfe man immer durch Aspiration, ob die Kanüle nicht irrtümlich intravasal liegt. 


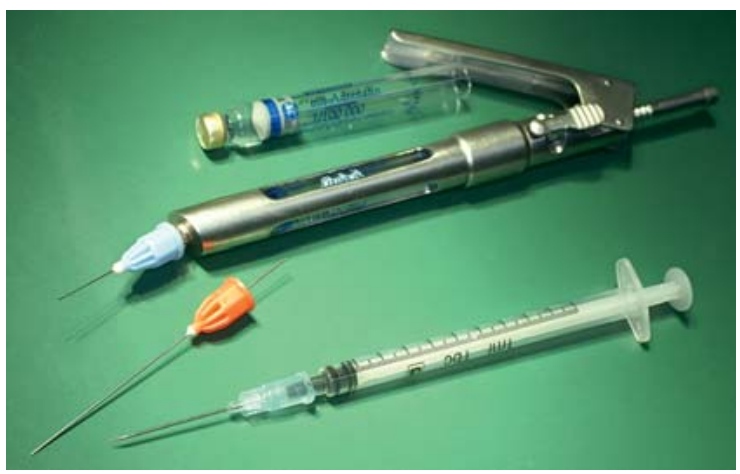

- Abb. 1 Unten im Bild eine Tuberkulinspritze mit Spardorn und blauer Kanüle (Durchmesser 0,6 mm). Darüber eine zahnärztliche Repetierspritze mit aufgesetzter Dentalnadel (Durchmesser 0,3 mm). Daneben eine Wechselampulle mit Lokalanästhetikum und eine lange Dentalnadel. Beachte die Doppelnadel der Dentalkanüle, die sich beim Einschrauben durch die Gummimembran der Ampulle sticht. (C) Stefan Gabriel

Eine praktische Alternative sind spezielle Zahnarztspritzen (zahnärztliche Zylinderampullen-Spritze) oder eine spezielle automatische Dosierspritze (z. B. Paroject ${ }^{\circledR}$ oder Nachbauten mit speziellen extrem dünnen Dentalnadeln). Diese geben als Repetierspritze ( $\bullet$ Abb. 1 ) bei jedem Klick des Hebelmechanismus eine definierte Menge Lokalanästhetikum unter hohem Druck, aber mit sehr kleinem Volumen $(0,06 \mathrm{ml})$ ab. Die Nadeln sind so dünn (30 Gauge, 0,3 mm Durchmesser), dass sie in den Parodontalspalt eingeführt werden können (sog. intraligamentäre Injektion) oder auch den Knochen und die feste Gingiva penetrieren können zur lokalen Infiltration.

Zahnärztliche Repetierspritzen sind sehr handlich und zur parodontalen Infiltration wie zur Nervenblockade hervorragend geeignet. Der Autor benutzt sie in seiner Praxis ausschließlich auch zur Leitungsanästhesie am Kopf. Die unbeabsichtigte Applikation einer zu großen Dosis ist damit praktisch unmöglich. Angebrochene Zylinderampullen können weiter verwendet und die Einmalnadeln jederzeit ausgewechselt werden.

Lokalanästhesien am Kopf:

- Lokalanästhesie

- Oberflächenanästhesie, z.B. Kornea, Konjunktiva, Kehlkopf

- lokale Infiltration

- intraligamentäre Infiltration

- Leitungsanästhesie

- Leitungsblock - „unterer“ oder rostraler Block

- Leitungsblock - „oberer“ oder kaudaler Block

Die sensiblen Nerven des Kopfes sind Äste des N. trigeminus, die im Oberkiefer als N. maxillaris/N. infraorbitalis und im Unterkiefer als $\mathrm{N}$. mandibularis/N. mentalis 


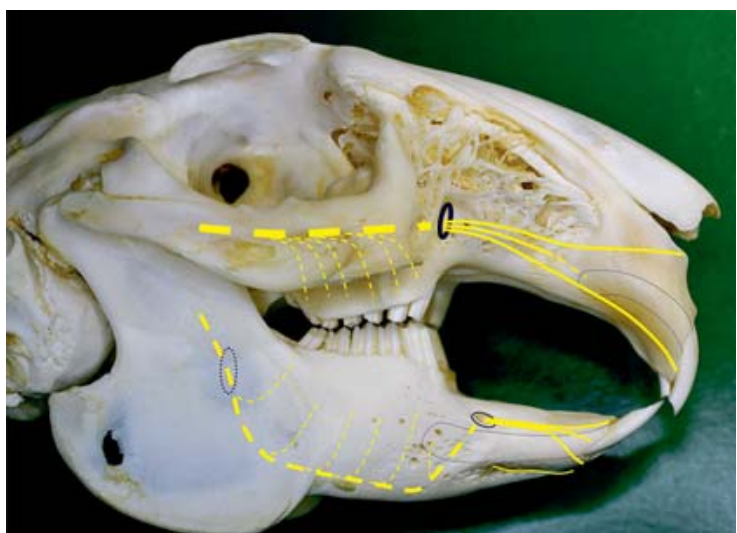

- Abb. 2 Schematischer Verlauf der relevanten Kopfnerven am Kaninchenschädel mit den Austrittsöffnungen. Das punktiert gezeichnete Foramen mandibulare liegt medial im Unterkiefer, die gestrichelten Bahnen verlaufen im Kieferkanal. @ Stefan Gabriel

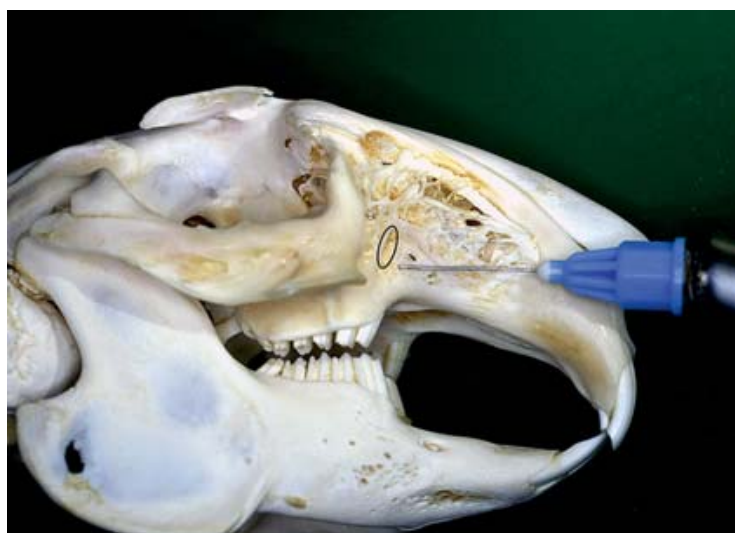

- Abb. 3 Rostraler Block des N. infraorbitalis am Austritt aus dem Foramen infraorbitale. Demonstration der Injektionsrichtung am Kaninchenschädel: Subkutanes Vorschieben der Nadel tangential zur seitlichen Nasenwand. (c) Stefan Gabriel in ihrem Verlauf hoch oder tief geblockt werden können ( $\triangleright$ Abb. 2). Der Verlauf der Kopfnerven ist grundsätzlich tierartübergreifend ähnlich, allerdings bestehen durch die engen räumlichen Verhältnisse und die unterschiedliche Lage der Zahnwurzeln erhebliche speziesspezifische Besonderheiten, die dargestellt werden. Durch eine hohe Blockade, sog. kaudaler Block, können ganze Kieferquadranten ausgeschaltet werden. In der Praxis spielen jedoch lediglich die distalen Blockaden, nämlich der rostrale Mandibularblock und der Block des $\mathrm{N}$. infraorbitalis eine Rolle. Der kaudale Mandibularblock durch Ausschalten des N. mandibularis vor seinem Eintritt in den Mandibularkanal medial am Foramen mandibulare ist grund- sätzlich auch möglich. Er ist allerdings technisch schwierig zu setzen und hat den Nachteil, dass eine stundenlange Zungenlähmung eintreten kann, unter der sich die Patienten fatale Verletzungen der Zunge zuziehen oder sich verschlucken können.

\section{Oberkiefer}

Der N. infraorbitalis kann direkt an seinem Austritt am Foramen infraorbitale blockiert werden. Beim Kaninchen ( $\triangleright$ Abb. 3) ist das Foramen gut palpierbar und liegt auf der Maxilla rostral des Arcus zygomaticus. Wenn man es horizontal über den seitlichen Nasenrücken ansticht, muss man darauf achten, tangential der Maxilla zu blei-
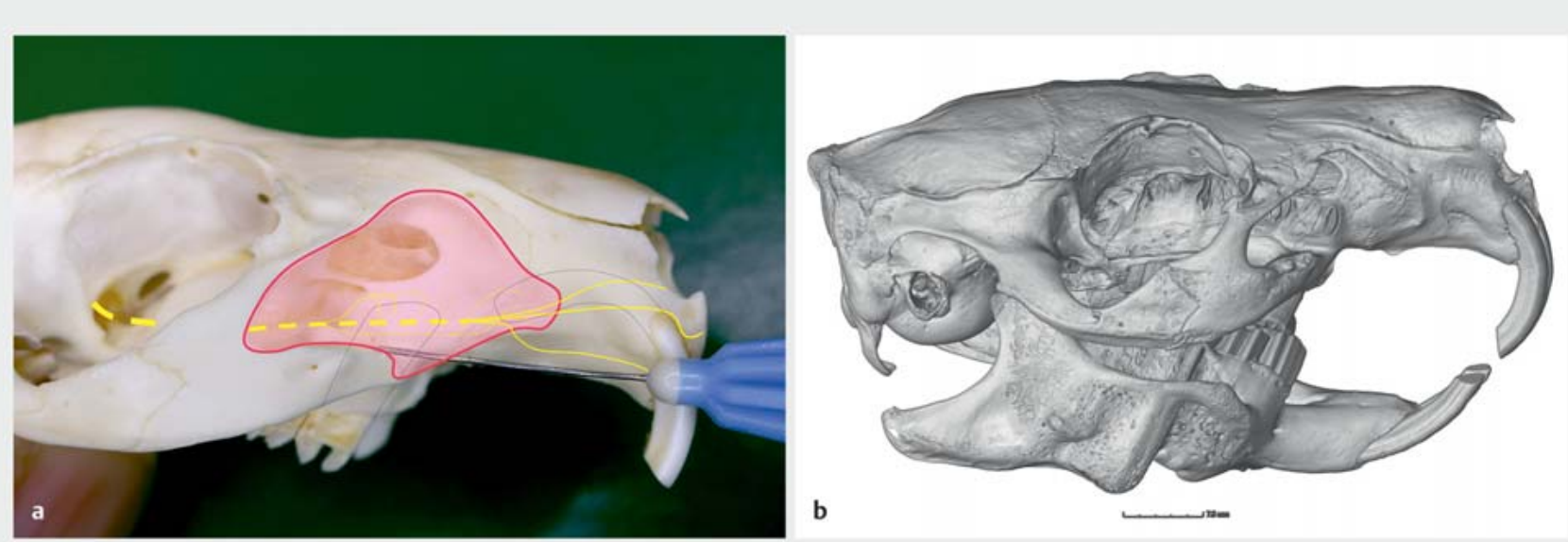

- Abb. 4 Rostraler Block des N. infraorbitalis in der infraorbitalen Rinne beim Meerschwein. a Die weite Öffnung des Foramen infraorbitale beim Meerschwein ist von der rostralen Portion des M. masseter bedeckt. Der N. infraorbitalis verläuft in einer Rinne am Boden des Kanals. b 3D-Rekonstruktion, hochauflösendes Mikro-CT eines Meerschweinchenschädels. Beachte den Verlauf der infraorbitalen Rinne ventral der Wurzelregion des Prämolaren im Oberkiefer. @ Stefan Gabriel 


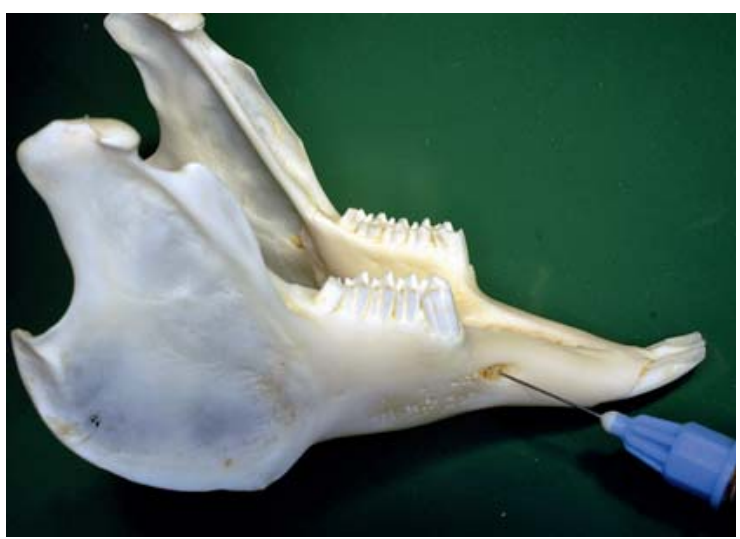

- Abb. 5 Der rostrale Mandibularblock durch Blockade der $\mathrm{Nn}$. mentales am Foramen mentale beim Kaninchen. (c) Stefan Gabriel

ben und nicht irrtümlich durch den trabekulären Knochen in die Nasenhöhle zu gelangen. Der N. infraorbitalis innerviert die Prämolaren, die Inzisiven, die Oberlippe und den vorderen Gaumen.

Bei den Caviomorphen (Meerschweinchen, A Abb. 4, Chinchilla) ist das große Foramen infraorbitale durch die infraorbitale Portion des mächtigen M. masseter bedeckt. Der Nervenverlauf in einer Rinne auf dem Boden der weiten Fossa infraorbitalis kann nur blind durch eine von rostral auf dem Periost tangential vorgeschobene Kanüle aufgesucht werden. Durch weiteres Vorschieben der Nadel können auch der Prämolar und der erste Molar anästhesiert werden.

\section{Unterkiefer}

Der rostrale Mandibularblock durch Blockade des $\mathrm{N}$. mentalis am Foramen mentale ist technisch einfach zu applizieren ( $\boldsymbol{A} \mathbf{A b b} \mathbf{5} \mathbf{5}$ und $\mathbf{6}$ ). Er wird zur Schmerzausschaltung an den Unterlippen und den Inzisiven verwendet. Dazu wird das Foramen mentale aufgesucht und die austretenden $\mathrm{Nn}$. mentales mit einem periostnahen Depot blockiert. Es kann mit einer dünnen Nadel auch direkt angestochen werden. Damit ist eine teilweise retrograde Injektion in den Mandibularkanal zur Anästhesie der Prämolaren und Inzisivenwurzeln möglich. Die Invisivenwurzel liegt bei Nagern allerdings so weit distal auf Höhe der Molaren, dass eine reine Leitungsanästhesie am Foramen mentale nicht den gesamten Inzisivus blocken kann. Hier ist die intraligamentäre Technik vermutlich effektiver ( A Abb. 7).

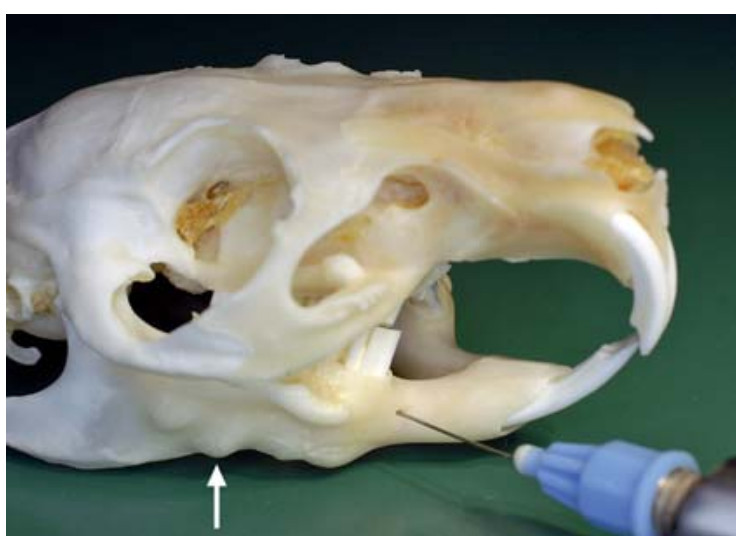

- Abb. 6 Der rostrale Mandibularblock durch Blockade des $\mathrm{N}$. mentalis am Foramen mentale beim Meerschwein. Beachte, dass die Innervation der Inzisivi nicht erreicht werden kann, weil die Inzisivenwurzeln auf Höhe der 2. Molaren liegen (Pfeil). @ Stefan Gabriel

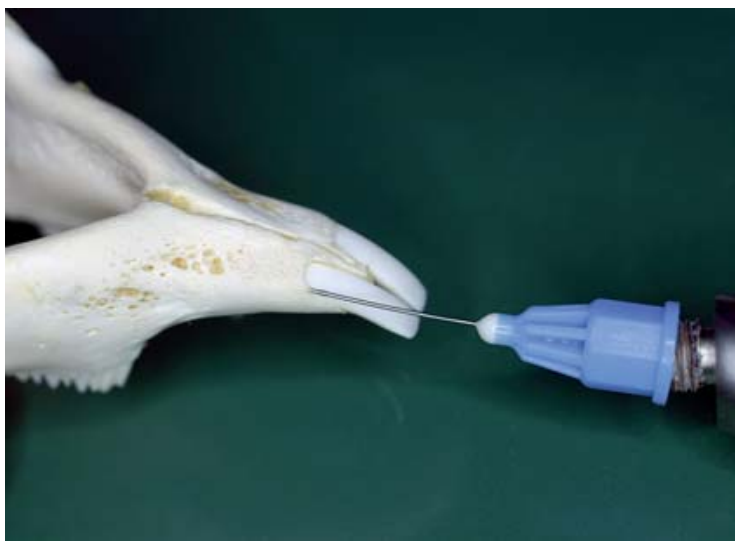

Abb. 7 Intraligamentäre Injektion in den Alveolarspalt des großen Inzisivus beim Kaninchen. Beachte, dass sich die dünne Dentalkanüle bis in die knöcherne Alveole vorschieben lässt. Die Nn. mentales sind von dieser Position aus durch subkutanes Vorschieben nach lateral erreichbar. @ Stefan Gabriel

Korrespondenzadresse

\section{Dr. Stefan Gabriel}

Tierärztliche Praxis für Kleintiere Dr. Stefan Gabriel Le-Puy-Straße 13 59872 Meschede tierarzt@drgabriel.de

\section{Bibliografie}

DOI https://doi.org/10.1055/s-0044-100874

kleintier konkret 2018; 21: 22-25

(c) Georg Thieme Verlag KG Stuttgart · New York

ISSN 1434-9132 